\title{
Development of a lipopolysaccharide (LPS)-supplemented adjuvant and its effects on cell-mediated and humoral immune responses in male rats immunized against sperm
}

\author{
Junko NOGUCHI ${ }^{1)}$, Shinya WATANABE ${ }^{2}$, Thanh Q. Dang NGUYEN"1), Kazuhiro KIKUCHI ${ }^{1)}$ and \\ Hiroyuki KANEKO ${ }^{1)}$
}

\author{
1) Institute of Agrobiological Sciences, National Agriculture and Food Research Organization (NARO), \\ Ibaraki 305-8602, Japan \\ 2) Institute of Livestock and Grassland Science, NARO, Ibaraki 305-0901, Japan
}

\begin{abstract}
Supplementation with lipopolysaccharide (LPS) from non-pathogenic Escherichia coli was found to enhance the adjuvant effects of a veterinary vaccine adjuvant (ISA $71 \mathrm{VG}^{\circledR}$ ). Sperm immunization using $71 \mathrm{VG}$ as an adjuvant in the immature period induced infertility in $25 \%$ of male rats, whereas this increased to $62.5 \%$ after immunization with $71 \mathrm{VG}+\mathrm{LPS}$ or Freund's complete adjuvant (FCA). Mean testicular weight of non-sterile males in the 71VG + LPS group was significantly lower than that in the 71VG or FCA group. Histological examination of testicular tissue from sterile males demonstrated severe impairment of spermatogenesis due to experimental autoimmune orchitis, a cell-mediated autoimmune condition. The serum anti-sperm titer was elevated in the three sperm-immunized groups relative to male rats treated with adjuvant alone, but the titer was higher in the 71VG + LPS and FCA groups than in the 71VG group. We consider that this LPS-supplemented adjuvant stimulates both humoral and cell-mediated immune responses to an extent comparable to FCA.
\end{abstract}

Key words: Adjuvant, Autoimmune orchitis, Lipopolysaccharide (LPS), Rat, Sperm immunization

(J. Reprod. Dev. 63: 111-115, 2017)

$\mathbf{F}$ ertility control is a non-lethal approach for reducing the population of overabundant wildlife. Gonadotropin releasing hormone and porcine zona pellucida based immunocontraceptive vaccines are already in practical use in the United States of America [1]. Successful immunocontraception has been achieved with these two antigens through combination with a veterinary adjuvant, AdjuVac ${ }^{\circledR}$. This powerful adjuvant was developed by modifying a mycoplasma vaccine, Mycoper ${ }^{\circledR}$, and was proven not to cause inflammation at the injection site [2]. However, its use in Japan is probably impermissible, since AdjuVac contains killed bacteria (Mycobacterium spp.) thus contravening the domestic Policy for Control of Infectious Diseases in Domestic Animals. Freund's complete adjuvant (FCA) is a strong adjuvant containing Mycoplasma spp., but it was proven to cause inflammation at the injection site [2], thus making it suitable only for experimental use in terms of animal welfare. With the long-term aim of achieving effective immunocontraception for density control of wildlife in Japan (e.g., sika deer [3]), the present study was undertaken to develop an alternative adjuvant that would overcome the two problems described above, allowing its registration as a vaccine adjuvant for field use. To achieve efficacy and gain public acceptance, we investigated the effects of adding non-pathogenic

Received: October 12, 2016

Accepted: November 7, 2016

Published online in J-STAGE: November 28, 2016

(C)2017 by the Society for Reproduction and Development

Correspondence: J Noguchi (e-mail: jgucci@affrc.go.jp)

This is an open-access article distributed under the terms of the Creative Commons Attribution Non-Commercial No Derivatives (by-nc-nd) License $<$ http://creativecommons.org/licenses/by-nc-nd/4.0/>.
Escherichia coli lipopolysaccharide (LPS) to montanide ISA 71 VG ${ }^{\circledR}$, a mineral oil-based water-in-oil-type veterinary vaccine adjuvant. The source and amount of LPS were based on data in previous reports [4, 5]. LPS is a structural component of the outer membrane of Gramnegative bacteria. It consists of three major domains: O-specific chain, core, and lipid A. Lipid A binds to Toll-like receptor 4 on immune cells to activate both innate and adaptive immune responses [6]. A derivative of LPS, mono-phosphoryl lipid A, has been approved as a human vaccine adjuvant [7].

A historic study by Freund et al. in 1953 showed that administration of testis-derived antigens or sperm emulsified in FCA was able to induce experimental autoimmune orchitis (EAO) in guinea pigs [8]. Later, methods to induce EAO in mice $[9,10]$ and rats $[11,12]$ were developed with some modification of the immunization procedure, notably intravenous (i.v.) injection of killed Bordetella pertussis to enhance the adjuvant effect of FCA. Subsequent studies involving transfer of $\mathrm{T}$ cells from immunized males to syngeneic recipients revealed that the EAO was a result of cell-mediated immunity [13-16], CD4 ${ }^{+} \mathrm{T}$ cells in particular playing a leading role [17]. Our preliminary experiments showed that it was possible to induce EAO in rats by immunization in the immature period with sperm emulsified in FCA, without subsequent injection of Bordetella pertussis. After several trials with different doses of sperm, we found that two inoculations of $2 \times 10^{7}$ epidydimal sperm emulsified in FCA induced $\mathrm{EAO}$ in over $50 \%$ of recipients with a concomitant increase in the serum level of anti-sperm IgG. Therefore, we considered it would be feasible to evaluate the adjuvant's effectiveness in terms of both cell-mediated and humoral immune responses by replacing FCA in our immunization program. 
Table 1. Effects of sperm inoculation on fertility in male rats

\begin{tabular}{|c|c|c|c|c|c|c|c|c|c|c|}
\hline \multicolumn{2}{|l|}{ Treatment* } & \multirow[b]{2}{*}{$\mathrm{n}$} & \multicolumn{2}{|c|}{ Fertility ${ }^{* *}(10$ weeks of age) } & \multicolumn{3}{|c|}{ Fertility $* *$ ( 20 weeks of age $)$} & \multicolumn{3}{|c|}{ Testis weight $^{* * *}(\mathrm{~g})$} \\
\hline adjuvant & sperm & & $/$ all $^{\dagger}$ & / fertile ${ }^{\dagger \dagger}(\mathrm{n})$ & /all ${ }^{\dagger}$ & $/$ fertile $^{\dagger \dagger}$ (n) & $\begin{array}{l}\text { sterile males } \\
\quad(\%)\end{array}$ & $/ \mathrm{all}^{\dagger}$ & $/$ fertile (n) ${ }^{\dagger}$ & / sterile (n) ${ }^{\dagger}$ \\
\hline none & - & 4 & $16.0 \pm 0.50$ & $16.0 \pm 0.50$ & $14.1 \pm 0.34^{\mathrm{ab}}$ & $14.1 \pm 0.34(4)^{\mathrm{a}}$ & $0 / 4(0)$ & $1.51 \pm 0.024^{\mathrm{a}}$ & $1.51 \pm 0.024(4)^{\mathrm{a}}$ & - \\
\hline FCA & - & 4 & $16.4 \pm 1.38$ & $16.4 \pm 1.38$ & $14.8 \pm 0.63^{\mathrm{a}}$ & $14.8 \pm 0.63(4)^{\mathrm{a}}$ & $0 / 4(0)$ & $1.43 \pm 0.034^{\mathrm{a}}$ & $1.43 \pm 0.034(4)^{\mathrm{ab}}$ & - \\
\hline ISA 71VG + LPS & - & 4 & $14.4 \pm 0.80$ & $14.4 \pm 0.80$ & $15.0 \pm 0.84^{\mathrm{a}}$ & $15.0 \pm 0.84(4)^{\mathrm{a}}$ & $0 / 4(0)$ & $1.59 \pm 0.007^{\mathrm{a}}$ & $1.59 \pm 0.007(4)^{\mathrm{a}}$ & - \\
\hline FCA & + & 8 & $13.3 \pm 1.94$ & $15.2 \pm 0.42(7)$ & $4.8 \pm 2.50^{\mathrm{bc}}$ & $14.2 \pm 1.42(3)^{\mathrm{a}}$ & $5 / 8(62.5)$ & $0.83 \pm 0.100^{\mathrm{b}}$ & $1.20 \pm 0.123(3)^{\mathrm{ab}}$ & $0.60 \pm 0.018$ \\
\hline ISA $71 \mathrm{VG}$ & + & 4 & $10.1 \pm 3.38$ & $13.5 \pm 0.00$ & $11.0 \pm 3.67^{\mathrm{ac}}$ & $14.7 \pm 0.33(3)^{\mathrm{a}}$ & $1 / 4(25.0)$ & $1.12 \pm 0.229^{\mathrm{a}}$ & $1.34 \pm 0.186(3)^{\mathrm{ab}}$ & $0.48(1)$ \\
\hline ISA 71VG + LPS & + & 8 & $11.0 \pm 2.43$ & $14.7 \pm 0.56(6)$ & $3.1 \pm 1.61^{\mathrm{c}}$ & $8.3 \pm 1.59(3)^{b}$ & $5 / 8(62.5)$ & $0.76 \pm 0.062^{b}$ & $0.94 \pm 0.094(3)^{\mathrm{b}}$ & $0.65 \pm 0.046(5)$ \\
\hline
\end{tabular}

* Each male was administered adjuvant with/without sperm $\left(2 \times 10^{7}\right)$ at 2 and 4 weeks of age. ** Fertility was examined by mating tests. Each male was placed with a pro-estrous female and the female was examined for the number of fetuses at 12-14 days after mating. *** Testicular weights were measured at 21-22 weeks of age, and a few at 30 weeks of age. ${ }^{\dagger}$ Means \pm SEM were calculated from numbers of males in column " $n " . \dagger$ Means \pm SEM were calculated from the numbers of fertile males indicated in parentheses. ${ }^{\mathrm{a}-\mathrm{c}}$ Values with different superscripts are significantly different $(\mathrm{P}<0.05)$.

In order to evaluate the effects of adjuvants on the induction of EAO, we examined the fertility of immunized males at 10 and 20 weeks of age by mating them with intact female rats. The results are shown in Table 1. All of the males examined showed normal libido and copulated with pro-estrous females. Next morning vaginal plugs were detected in all of the mated female rats. There was no significant difference in the number of implantations among 3 control groups (non-treated, treated with FCA, or treated with LPS-supplemented ISA $71 \mathrm{VG}$ ), indicating that these adjuvants do not intrinsically interfere with male fertility. One out of 8 male rats immunized with sperm emulsified in FCA (referred to hereafter as sperm+FCA) was infertile at 10 weeks of age. This male and 4 additional males in the group were sterile at 20 weeks of age, while the other 3 males remained fertile with the number of implantations being comparable to those in the 3 control groups. In the group treated with sperm and ISA 71VG supplemented with LPS (referred to hereafter as sperm $+71 \mathrm{VG}+\mathrm{LPS}), 2$ out of 8 rats were sterile at 10 weeks of age, and these 2 and an additional 3 males exhibited sterility at 20 weeks of age. The mean number of implantations for non-sterile males in this group $(8.3 \pm 1.59, \mathrm{n}=3$, mean \pm SEM) was significantly lower than that in any of the other groups, indicating these 3 males were sub-fertile. In contrast, treatment with sperm and ISA 71VG (referred to hereafter as sperm $+71 \mathrm{VG}$ ) rendered 1 out of 4 males sterile without decreasing the number of implantations for the other 3 fertile males. We examined testicular weight and morphology in all of the treated males to clarify the prevalence of EAO. There was no significant difference in testicular weight between the non-treated and the two control groups, and no aberrant morphological features were evident in the testicular tissue (Table 1, Fig. 1A). These data suggested that administration of FCA and ISA 71VG supplemented with LPS (the latter being referred to as $71 \mathrm{VG}+$ LPS hereafter) in the immature period had no influence on spermatogenesis after maturation. Mean testicular weight was significantly lower in the sperm + FCA and sperm $+71 \mathrm{VG}+$ LPS groups than in the control and sperm $+71 \mathrm{VG}$ groups. However, it was noteworthy that the testicular weight of fertile males in the 3 sperm-immunized groups was relatively lower than that in the control groups, the sperm + $71 \mathrm{VG}+$ LPS group in particular showing a significantly lower value than that in the non-treated group. In these smaller testes, some of the seminiferous tubules showed loss or partial depletion of germ cells (Fig. 1B). The testes of sterile males in the 3 immunized groups showed bilateral atrophy, the weight being $0.44-0.67 \mathrm{~g}$, i.e., less than half of that in the 3 control groups. Histological examination of these testes demonstrated EAO with severe impairment of spermatogenesis, resulting in complete loss of differentiated germ cells (Fig. 1C and D). It was noteworthy that marked morphological changes were not evident in the interstitial tissues of the testis, despite the marked damage to seminiferous tubules. Previous studies of EAO had shown that immunization with testicular antigen induced infiltration of considerable amounts of immune cells into the testicular interstitial tissue of mice [10] and rats [11, 12]. These immune cells were shown to produce pro-inflammatory cytokines, such as TNF- $\alpha$ and interferon- $\gamma$, which are thought to damage germ cells directly or through modification of Sertoli cell function [17, 18]. The impaired spermatogenesis we observed here, in the absence of marked inflammatory cell infiltration, does not seem to match this type of pathogenesis. In the affected testis of 3 immunized groups, small number of TNF- $\alpha$-positive cells attaching to the seminiferous tubules were detected (data not shown). These cells might influence Sertoli cell functions, resulting in impaired spermatogenesis. Further investigations are ongoing to clarify the mechanism responsible for spermatogenesis impairment without accumulation of immune cells.

Next, we examined serum titers of anti-sperm antibody to evaluate the effects of adjuvant on humoral immunity. The non-treated group showed a very slight increase in the serum anti-sperm titer as the rats aged (Fig. 2A), the highest value $\left(3.2 \times 10^{3} \mathrm{cps}\right)$ being detected at 20 weeks of age. The titers in the $71 \mathrm{VG}+$ LPS group were indistinguishable from those in the non-treated group. In the FCA-treated group, however, the mean titer was higher than in the non-treated and 71VG + LPS groups after 10 weeks of age, and the difference between this and the other two control groups was significant at 15 weeks of age. Relatively higher titers in 2 out of 4 rats in the FCA-treated group $\left(23.7\right.$ and $\left.27.7 \times 10^{3} \mathrm{cps}\right)$ reflected the mean titer at 20 weeks of age $\left(14.3 \pm 6.7 \times 10^{3} \mathrm{cps}\right)$. The reason for this increase was unclear. Establishment of the humoral immune reaction against sperm was reflected as elevated titers in all of the 3 sperm-immunized groups. In the sperm + FCA group, individual titers ranged between 30.4 and $329.0 \times 10^{3} \mathrm{cps}$ at 8 weeks of age, being at least 20-fold higher than those in the FCA-treated group (Fig. 2C), where the average count was $1.5 \pm 0.4 \times 10^{3} \mathrm{cps}$ at the 

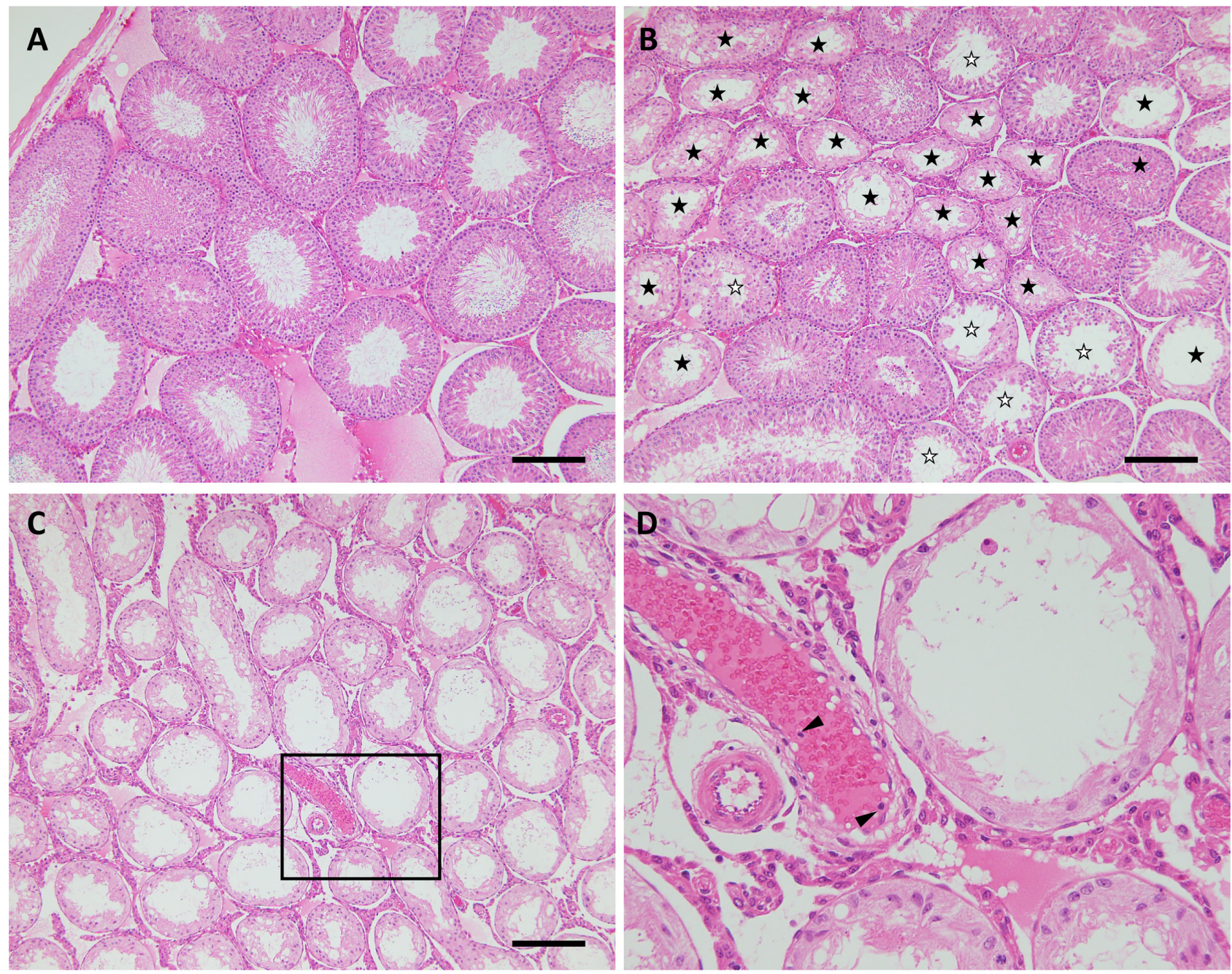

Fig. 1. Testicular morphology of sperm-immunized rats. Control males (A) administered 71VG+LPS showed normal spermatogenesis. The prevalence of autoimmune orchitis was observed in males immunized with sperm emulsified in 71VG + LPS. In cases of moderate orchitis (B), seminiferous tubules with partial $(\hat{\zeta})$ or almost complete loss of germ cells $(\star)$ were observed adjacent to normal tubules. In cases of severe orchitis (C and D), almost all of the tubules had lost germ cells. Inflammatory cells were observed in the blood vessels (arrowheads), but not in the interstitial tissue. The box in C is magnified (D). Scale bar $=100 \mu \mathrm{m}$.

same age. Individual peak titers in the sperm + FCA group ranged between 117.9 and $418.0 \times 10^{3} \mathrm{cps}$ at 10 or 15 weeks of age, and a declining tendency was detected at 20 weeks of age. In the sperm + $71 \mathrm{VG}$ group, individual titers ranged between 69.5 and $204.1 \times 10^{3}$ cps at 8 weeks of age, increasing slightly thereafter with individual peaks of between 121.3 and $236.3 \times 10^{3} \mathrm{cps}$ (Fig. 2B). These results indicate that $71 \mathrm{VG}$ activated the humoral immune response moderately in comparison with FCA. Enhancement of the immune response by LPS supplementation was clearly evident in the sperm $+71 \mathrm{VG}$ + LPS group. The titers at 8 weeks of age ranged from 133.0 to $390.1 \times 10^{3} \mathrm{cps}$, and at this point the titer reached individual peaks in 2 out of 8 rats; in the other 6 rats the titers continued to increase until 15 weeks of age, reaching individual peaks of between 182.9 and $477.4 \times 10^{3} \mathrm{cps}$ (Fig. 2D). Endogenous antigens produced in parallel with spermatogenesis might have stimulated the memory cells generated by immunization during the immature period, the activated immune cells then producing the $\mathrm{IgG}$ we detected in 3 sperm-immunized groups. Although the blood-testis barrier in the seminiferous epithelium as well as the tight junctions formed in the epithelium of the efferent and epididymal duct separate sperm antigens from the immune system, it has been reported that there are some specific regions where the barrier is not complete [17]. Discordance of individual reproductive abilities and levels of serum anti-sperm antibody titer in the immunized groups suggests that cell-mediated immunity was fundamentally involved in this disorder [13-16]. The results might also suggest different autoantigenicity of sperm antigens in the humoral and cell-mediated immune response. It is possible that autoimmune orchitis is induced by the immune cells activated with orchitogenic autoantigens among sperm antigens; therefore, we started experiments for identification of sperm antigens 
A
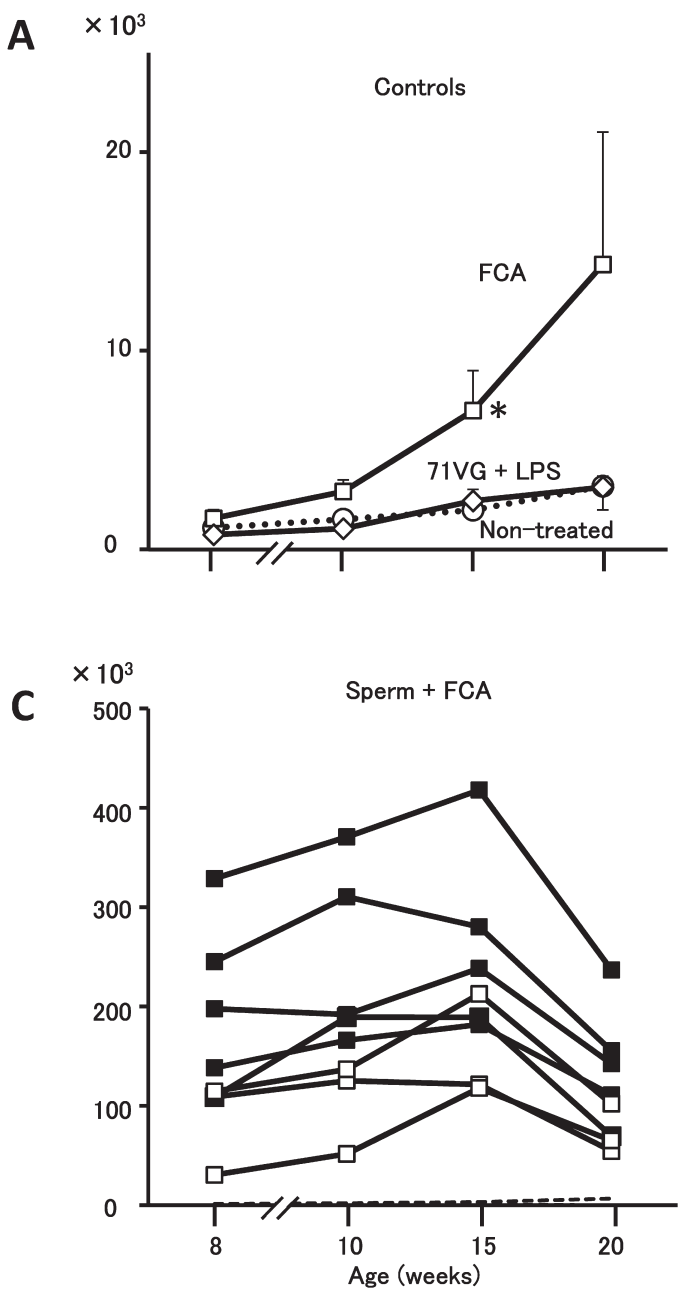

B $\times 10$

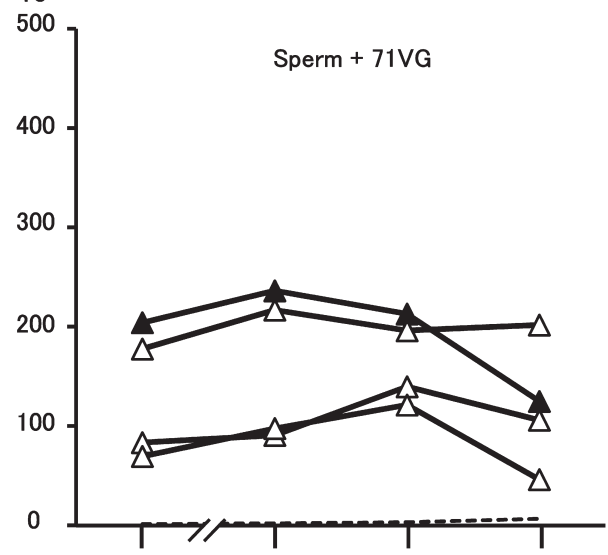

$\times 10^{3} \quad$ Sperm $+71 \mathrm{VG}+$ LPS

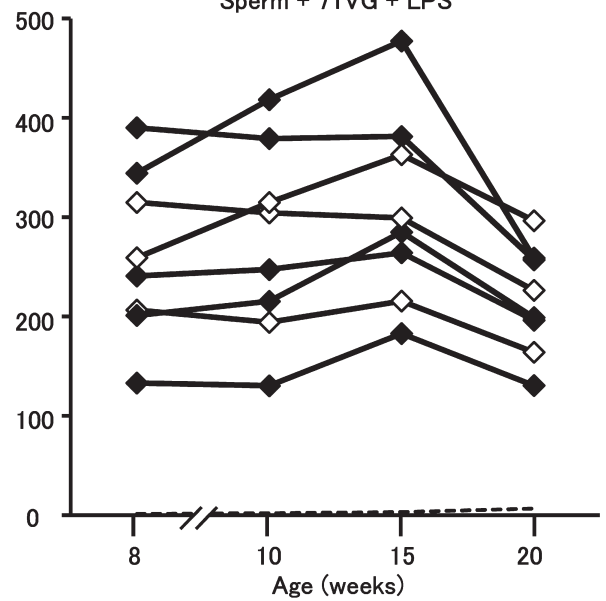

Fig. 2. Anti-sperm antibody titers in serum of male rats after immunization with sperm and adjuvant in the immature period. Values are shown as counts per second (cps). In the control groups (A), means \pm SEM are shown. Non-treated $(\circ)$ and FCA ( $\square)$ and ISA 71VG + LPS $(\diamond)$ groups. In the FCAtreated group, a significant difference from the other two groups was detected at 15 weeks of age. In the sperm-immunized groups (B, C and D), individual data are shown. Rats were immunized with sperm and ISA 71VG (B), sperm and FCA (C) or sperm and ISA 71VG supplemented with LPS (D). Data for males that were sterile at 20 weeks of age are shown with filled markers. Dashed lines in B, C and D show mean $+3 \times$ SEM of the non-treated group.

causative of the orchitis.

In conclusion, it is evident that the effectiveness of montanide ISA $71 \mathrm{VG}$, a commercial veterinary vaccine adjuvant, for activating the immune response can be enhanced by LPS supplementation. With regard to safety, we did not note any abnormal signs during the experiments, for example injection site inflammation, weight loss or other external features. However, further verification of the safety of this adjuvant will be needed.

\section{Methods}

\section{Immunization and sample collection}

All the rats used (Iar: Wistar-Imamichi strain) were purchased from the Institute of Animal Reproduction (Kasumigaura, Japan) and maintained in the Institute of Agrobiological Sciences, NARO. All of the experimental procedures were approved by the Committee on the Care and Use of Experimental Animals at the Institute of Agrobiological Sciences, NARO.

Epididymal sperm were collected from the cauda epididymis of adult males, divided into small aliquots, and stored at $-80^{\circ} \mathrm{C}$ until use. For immunization, the thawed sperm were suspended in saline. LPS (from E.coli O127:87, Sigma, St. Louis, USA) was added if necessary at $0.1 \mathrm{mg} / \mathrm{kg} \mathrm{BW}$. The suspension was emulsified in an equal volume of FCA (Wako, Osaka, Japan) or montanide ISA 71VG (a gift from Seppic, Paris, France). Immature rats at 12-14 days of age were divided into 6 groups: non-treated, treated with adjuvant alone (FCA or 71VG + LPS), and 3 sperm-immunized groups with FCA, 71VG or 71VG + LPS. Rats were injected with $100 \mu$ of the emulsion including $2 \times 10^{7}$ sperm subcutaneously in the back under light ether anesthesia. The second immunization with $200 \mu$ of the 
emulsion including $2 \times 10^{7}$ sperm was performed 2 weeks later. Controls were administered an emulsion of saline and adjuvant. Blood samples were collected from the jugular vein under ether anesthesia at 8,10,15 and 20 weeks of age. Fertility of the treated males was examined at $10-11$ and 20-22 weeks of age by mating tests; each male was mated with an adult female rat at pro-estrus overnight. Successful mating was confirmed by the presence of vaginal plugs the following morning. Females were examined for implantation between 12 and 14 days after mating. For the mating test, each male was tested at least twice with an interval of 3-4 days. Testes were collected from the males at 21-22 weeks of age, and at 30 weeks in some cases. After being weighed, the testes were fixed in Bouin's fixative. Paraffin sections were prepared and stained with hematoxylin and eosin for morphological examination.

\section{Determination of anti-sperm antibody titer}

The anti-sperm antibody titer in serum was determined as follows. Antigens extracted from the sperm were adsorbed onto the wells of a 96-well plate (FluoroNunc, Thermo Fisher Scientific, Waltham, MA, USA) at a concentration of $5 \mu \mathrm{g} / \mathrm{ml}$ (total protein concentration determined by Bradford assay) with coating buffer [19] at $25^{\circ} \mathrm{C}$. The antigens used were prepared as follows. Epididymal sperm were suspended in $0.5 \%$ CHAPS (Dojindo, Kumamoto, Japan) PBS at a concentration of $10^{8}$ sperm per $\mathrm{ml}$. The suspension was frozen and thawed three times, centrifuged at $17,000 \times \mathrm{g}$ for $30 \mathrm{~min}$ at $4{ }^{\circ} \mathrm{C}$, and the supernatant was collected. Rabbit anti-rat IgG (Merck Millipore, Temecula, CA, USA) was labeled with an Eu-chelate of N1-(pisothiocyanatobenzyl)-diethylenetriamine-N1,N2,N3,N3-tetraacetic acid (Eu-Labeling reagent; Perkin Elmer, Waltham, MA, USA) by the methods described elsewhere [19]. Rat serum diluted 1:1000 was pipetted into antigen-coated wells, followed by incubation overnight at $25^{\circ} \mathrm{C}$. After the wells had been rinsed with washing buffer [19], the Eu-labeled antibody was added to each well $\left(2 \times 10^{6}\right.$ counts per second [cps] per $100 \mu \mathrm{l})$, and the wells were incubated for $3 \mathrm{~h}$. After the wells had been rinsed, $100 \mu$ of enhancement solution (Perkin Elmer) was added to each well, and the fluorescence in each well was measured with a fluorometer (Arvo, Perkin Elmer). Anti-sperm antibody titer was expressed as cps for each well.

\section{Statistical analysis}

Statistical analysis was performed using SAS add-in 6.1 for Microsoft ${ }^{\circledR}$ Office. When significance was detected by one-way ANOVA, the significance of differences in means among the groups was determined by Tukey's test $(\mathrm{P}<0.05)$.

\section{Acknowledgments}

We thank Ms Miwa Osaki and Mitsuru Nagai for technical as- sistance. This research was supported by the Science and Technology Research Promotion Program for Agriculture, Forestry, Fisheries and Food Industry (25026A) of the Ministry of Agriculture, Forestry and Fisheries of Japan.

\section{References}

1. Kirkpatrick JF, Lyda RO, Frank KM. Contraceptive vaccines for wildlife: a review. $\mathrm{Am}$ $J$ Reprod Immunol 2011; 66: 40-50. [Medline] [CrossRef]

2. Powers JG, Nash PB, Rhyan JC, Yoder CA, Miller LA. Comparison of immune and adverse effects induced by AdjuVac and Freunds complete adjuvant in New Zealand white rabbits (Oryctolagus cuniculus). Lab Anim (NY) 2007; 36: 51-58. [Medline] [CrossRef]

3. Noguchi J. Overabundance of Sika Deer and Immunocontraception. J Reprod Dev 2017; 63: $13-16$.

4. Gerdprasert O, OBryan MK, Muir JA, Caldwell AM, Schlatt S, de Kretser DM, Hedger MP. The response of testicular leukocytes to lipopolysaccharide-induced inflammation: further evidence for heterogeneity of the testicular macrophage population. Cell Tissue Res 2002; 308: 277-285. [Medline] [CrossRef]

5. OBryan MK, Schlatt S, Phillips DJ, de Kretser DM, Hedger MP. Bacterial lipopolysaccharide-induced inflammation compromises testicular function at multiple levels in vivo. Endocrinology 2000; 141: 238-246. [Medline]

6. Bohannon JK, Hernandez A, Enkhbaatar P, Adams WL, Sherwood ER. The immunobiology of toll-like receptor 4 agonists: from endotoxin tolerance to immunoadjuvants Shock 2013; 40: 451-462. [Medline] [CrossRef]

7. Sakai HK. Adjuvants. Rinsho To Uirusu 2013; 41: 1-9. (in Japanese).

8. Freund J, Lipton MM, Thompson GE. Aspermatogenesis in the guinea pig induced by testicular tissue and adjuvants. J Exp Med 1953; 97: 711-726. [Medline] [CrossRef]

9. Hargis BJ, Malkiel S, Berkelhammer J. Immunologically induced aspermatogenesis in the white mouse. J Immunol 1968; 101: 374-376. [Medline]

10. Kohno S, Munoz JA, Williams TM, Teuscher C, Bernard CCA, Tung KSK. Immunopathology of murine experimental allergic orchitis. J Immunol 1983; 130: 2675-2682. [Medline]

11. Doncel GF, Di Paola JA, Lustig L. Sequential study of the histopathology and cellular and humoral immune response during the development of an autoimmune orchitis in Wistar rats. Am J Reprod Immunol 1989; 20: 44-51. [Medline] [CrossRef]

12. Zhou ZZ, Zheng Y, Steenstra R, Hickey WF, Teuscher C. Actively-induced experimental allergic orchitis (EAO) in Lewis/NCR rats: sequential histo- and immunopathologic analysis. Autoimmunity 1989; 3: 125-134. [Medline] [CrossRef]

13. Tung KSK, Leong C, McCarty T. Pathogenesis of experimental allergic orchitis. III. T lymphocyte requirement in local adoptive transfer by peritoneal exudate cells. $J$ Immunol 1977; 118: 1774-1779. [Medline]

14. Mahi-Brown CA, Yule TD, Tung KSK. Adoptive transfer of murine autoimmune orchitis to naive recipients with immune lymphocytes. Cell Immunol 1987; 106: 408-419. [Medline] [CrossRef]

15. Feng ZY, Ming W, Li LD, Louis J, Fei WY. Adoptive transfer of murine autoimmune orchitis with sperm-specific T lymphoblasts. Arch Androl 1990; 24: 51-59. [Medline] [CrossRef]

16. Itoh M, Mukasa A, Tokunaga Y, Hiramine C, Hojo K. New experimental model for adoptive transfer of murine autoimmune orchitis. Andrologia 1991; 23: 415-420. [Medline] [CrossRef]

17. Yule TD, Tung KSK. Experimental autoimmune orchitis induced by testis and sperm antigen-specific $\mathrm{T}$ cell clones: an important pathogenic cytokine is tumor necrosis factor. Endocrinology 1993; 133: 1098-1107. [Medline]

18. Pérez CV, Theas MS, Jacobo PV, Jarazo-Dietrich S, Guazzone VA, Lustig L. Dual role of immune cells in the testis: Protective or pathogenic for germ cells? Spermatogenesis 2013; 3: e23870. [Medline] [CrossRef]

19. Kaneko H, Noguchi J, Kikuchi K, Todoroki J, Hasegawa Y. Alterations in peripheral concentrations of inhibin A in cattle studied using a time-resolved immunofluorometric assay: relationship with estradiol and follicle-stimulating hormone in various reproductive conditions. Biol Reprod 2002; 67: 38-45. [Medline] [CrossRef] 